\title{
Use of small angle neutron scattering to study the interaction of angiotensin II with model membranes
}

\author{
Julia Preu • Timo Jaeger • Vasil M. Garamus • \\ Thomas Gutberlet
}

Received: 28 July 2010/Revised: 30 December 2010/ Accepted: 6 January 2011/Published online: 3 February 2011

(C) European Biophysical Societies' Association 2011

\begin{abstract}
Understanding biological processes assumes a detailed understanding of the interaction of all involved molecules. Here the effect of the peptide hormone angiotensin II (Ang II), an agonist of the angiotensin receptors, on the structure of unilamellar and multilamellar dimyristoyl phosphatidylcholine vesicles was studied by small angle neutron scattering, dynamic light scattering and differential scanning calorimetry. The calorimetry data indicate a weak interaction of Ang II with the surface of the membrane bilayer, as the pretransition persists during all experiments, and the main transition is only slightly shifted towards higher temperatures. From the SANS data we were able to confirm the calorimetric data and verify the interaction of the hormone with the membrane surface. At low temperatures, when the lipid molecules are in the gel phase, more precisely in the ripple phase, the peptide penetrates in
\end{abstract}

J. Preu $(\bowtie)$

Niels Bohr Institute, Blegdamsvej 17,

2200 Copenhagen, Denmark

e-mail: Julia.Preu@mpibp-frankfurt.mpg.de

J. Preu - T. Jaeger · T. Gutberlet

Laboratory for Neutron Scattering, Paul Scherrer Institute,

Villigen PSI, Switzerland

Present Address:

J. Preu

Max-Planck Institute of Biophysics, Max-von Laue-Str. 3, 60438 Frankfurt, Germany

V. M. Garamus

Helmholtz-Zentrum Geesthacht Centre for Materials

and Coastal Research, Geesthacht, Germany

Present Address:

T. Gutberlet

Juelich Center for Neutron Science at FRM II,

Research Center Juelich, Garching, Germany the head group core, but due to the close packing of the acyl chains, the hydrophobic region is not affected. In a temperature region below but close to the region of the phase transition, the hydrophibic core starts to be affected by the peptide, and the same is true for the fluid phase. Upon binding of the peptide, the thickness of the head group increases, and the scattering length density of the head group starts to rise with increasing peptide concentrations. This interaction and binding to the membrane surface may be relevant for the relocation, binding and reconstitution of the angiotensin receptors into the membrane. Second, the peptide adsorption to the membrane surface may contribute to the binding of Ang II in the active site of the receptor.

Keywords Peptide-lipid interaction - Small angle neutron scattering · Angiotensin II - DMPC · Model membranes · Hypertension

\section{Introduction}

Hypertension is a growing problem worldwide, especially in the Western world. It is one of the major controllable risk factors associated with cardiovascular disease events such as myocardial infarcation, heart failure and end-stage diabetes (Pachori et al. 2001; Dézsi 2000; Labinjoh et al. 2000; Dimsdale et al. 1996; Neutel et al. 1999; MacMahon et al. 1990; WHO 2003).

In mammals, the blood pressure is regulated by the renin-angiotensin system, which plays a critical role in circulatory homeostasis. Part of this system is the peptide hormone angiotensin II (Asp-Arg-Val-Tyr-Ile-His-ProPhe), a potent vasoconstrictor that aids in blood pressure regulation as well as in body fluid balance maintenance. 
Ang II is derived from the precursor angiotensinogen by an enzymatic reaction catalyzed by renin and the angiotensin converting enzyme (ACE). In the heart, acting in both endocrine and paracrine fashion, Ang II regulates contractility, remodeling, growth and apoptosis, and reduces cell coupling and conduction velocity in cardiac muscles. The peptide acts on two major receptor subtypes, the Ang II type 1 receptor ( $\mathrm{AT}_{1}$ receptor) and the Ang II type 2 receptor $\left(\mathrm{AT}_{2}\right.$ receptor). Although both types of receptors were found in cardiac muscle cells, only the $\mathrm{AT}_{1}$ receptor was found to mediate classical cardiovascular functions like blood pressure elevation, vasoconstriction, aldosterone release, and renal absorption of water and sodium. From a pathophysiological point of view, like in renal diseases or hypertension, changes in the renin-angiotensin system occurred (Hong et al. 2008).

The role of Ang II in causing chronic diseases has been discussed in several publications (Ruiz-Ortega et al. 2003; Striker et al. 2008; Taylor 1999; de Gasparo et al. 2000). $\mathrm{ACE}$ inhibitors and $\mathrm{AT}_{1}$ receptor antagonists influence the cardiovascular status, demonstrating the important role of the renin-angiotensin system in physiological and pathophysiological states (Costerousse et al. 1992; Kurdi et al. 2005; NIH 2003). The inhibition of these enzymes (ACE) or $\mathrm{AT}_{1}$ receptors is believed to lower blood pressure. At present, the renin-angiotensin system has become a key target for drugs combating hypertension (Hong et al. 2008).

Hypertension can be treated by $\beta$-blockers, inhibiting the conversion of angiotensinogen to angiotensin I, or by specific inhibition of the $\mathrm{AT}_{1}$ receptor by an Ang II antagonist like Losartan with its active derivate E-3174. Apart from action on the membrane-bound receptor, an in vitro binding to lipids was described for Ang II, as well as Losartan. It was shown by ${ }^{1} \mathrm{H}-\mathrm{NMR}$ that the binding of Ang II depends on the $\mathrm{pH}$ of the surrounding medium and the temperature. Several amino acids of the peptide are involved in the binding to lipids (Valensin et al. 1986). Later, DSC and ${ }^{31}$ P-NMR were used to further characterize the interaction of DPPC with Ang II (Mavromoustakos et al. 1996). The phase behavior of the DPPC is affected by Ang II, as the main transition is shifted towards higher temperatures and the pre-transition is broadened.

Unfortunately, only little is known about the lipid environment the receptor is facing in vivo, especially if considering the changing lipid composition with respect to the tissue and the proliferation state. Moreover, lipid rafts, clathrin-coated pits and caveolae play an important role in the regulation of GPCRs (Chini and Parenti 2004). After syntheses in the endoplasmatic reticulum and glycosylation, the receptors are sorted to the plasma membrane (Leclerc et al. 2002; Wyse et al. 2003). Translocation into lipid rafts is often accompanied by agonist binding. Here, we demonstrate the binding of Ang II, an agonist of the AT receptors, on the example of phospholipid membranes using DMPC. With the main transition occurring at around $24^{\circ} \mathrm{C}$, it is possible to study the interaction in the gel respectively ripple phase, and fluid phase of the lipid without facing the problem of peptide degradation, which means the temperature-dependent destruction of the peptide.

To achieve a more complete view of the role of Ang II in blood pressure regulation, SANS, DSC and DLS were applied to study the interaction of the peptide Ang II with model membranes. DSC is a versatile tool in life science, allowing study of the thermotropic properties of a variety of materials and model systems (Bruylants et al. 2005). Especially for the study of membrane properties and changes, calorimetric techniques are a vital tool (Westh 2009). DSC was applied to study drug-lipid interactions as shown for vinorelbine (Koukoulitsa et al. 2006), malatonin (Severcan et al. 2005) and the calcium-dependent antibiotic daptomycin (Jung et al. 2008). Work performed with peptide-lipid interaction focuses on synthetic peptides and peptides with antimicrobial properties; examples are aurein, citropin and maculatin (Seto et al. 2007), maganin (Ludtke et al. 1996), PGLa (Pabst et al. 2008) and other cell-penetrating peptides (Alves et al. 2008).

SANS is a powerful technique to learn about the structure of a variety of materials. Basically all biological macromolecules and the interaction among them can be studied by SANS. Single proteins and complexes (Petoukhov and Svergun 2006; Svergun and Koch 2003) as well as polysaccharides like cyclodextrins (Burckbuchler et al. 2008) are studied (Wang et al. 2009). A variety of studies have dealt with lipids, their phases (Mason et al. 1999; Winter 2002) and the interaction with other classes of molecules. Bacterial membranes were used as sources (Mariani et al. 1997), but the majority of studies used synthetic lipids as a model system (Kucerka et al. 2007, 2008; Nagle and Tristram-Nagle 2000).

So far, bilayer thickness and interface areas have been studied (Balgavý et al. 2001; Pencer and Hallett 2000), as well as the multilamelarity, structure and hydration of different lipid systems (Schmiedel et al. 2006; Tung et al. 2008). In addition, contrast variation is a powerful tool to trace changes occurring upon mixing of different lipids (Knoll et al. 1981), such as changes in the bilayer thickness as a response to the addition of sterols (Pencer et al. 2005; Gallová et al. 2008; Kucerka et al. 2009), domain formation and rafts (Jacobson et al. 2007; Pencer et al. 2005).

The interaction of peptides and membranes was studied in combination with studies on cytotoxicity, like in the case of mellitin (Lundquist et al. 2008), and the development of new antimicrobial drugs (Huang 2000; Han et al. 2009). So far, no studies have been undertaken trying to study the 
interaction of peptides with membranes that do not lead to the formation of pores. Here, we show that it is possible to study the interaction of peptides with the membrane, even if the interaction does not disrupt the bilayer structure, but is based on a concentration-dependent adsorption process.

In addition to the SANS experiments, DLS experiments were performed. The light scattering provided a first insight into changes in the hydrodynamic radius upon addition of the peptide in comparison to a pure DMPC sample. The resulting hydrodynamic radius was used for the data fitting of the SANS studies.

\section{Methods}

\section{Materials}

1,2-Dimyristoyl-sn-glycero-3-phosphatidylcholine (DMPC) and the corresponding lipid with deuterated chains 1,2dimyristoyl-(d54)-sn-glycero-phosphocholine (d54-DMPC) were purchased from Avanti Polar Lipids, Inc. (Alabaster, AL). (4-(2-Hydroxyethyl) piperazine-1-ethanesulfonic acid sodium salt, (HEPES $99.5 \%$ pure), angiotensin II (human actetat, $93 \%$ pure), $\mathrm{NaCl}$ (analytical grade) and $\mathrm{D}_{2} \mathrm{O}(99.9 \%$ deuteration) were from Sigma-Aldrich. All chemicals were used without further purification.

\section{Differential scanning calorimetry}

DSC experiments were performed on a high sensitivity differential scanning calorimeter (Microcal VP-DSC, Northampton, MA), with a cell volume of $0.51 \mathrm{ml}$. The reference cell contained pure water, and the heating rate was $5^{\circ} \mathrm{C} / \mathrm{h}$. At least two calorimetric scans were performed with each sample to test reproducibility. Data evaluation was performed using a macro running with Igor Pro (WaveMetrics, Inc., Portland, $\mathrm{OR})$. The raw data were normalized by the lipid concentration, and the baseline was substracted. The phase transition temperatures were taken at peak values of the heat capacity, $\mathrm{c}_{p}$, and the calorimetric enthalpy $\Delta \mathrm{H}$ was calculated by integrating the peak areas. In addition, the position and area under the pre-transition peak were determined.

\section{Dynamic light scattering}

For the light scattering experiments, an ALV-5000 Multiple Tau Digital Correlator together with a HeNe-Laser at a wavelength of $632.8 \mathrm{~nm}$ was used. The temperature was controlled with an external heating circulator (F25-ME, Julabo, Seelbach, Germany). For aqueous solutions, a refractive index of 1.3320 was assumed, and the values for the viscosity varied according to the temperature change. The hydrodynamic radius was calculated as
$R_{h}=\frac{k T}{6 \pi \eta \Gamma} q^{2}$

with

$q=\frac{4 \pi n \sin \left(\frac{\theta}{2}\right)}{\lambda}$

$n$ as the refractive index, $\lambda$ the wavelength, $\theta$ the scattering angle, $k$ Boltzmann's constant, $\eta$ the solvent viscosity and $\Gamma$ the average decay rate.

Small angle neutron scattering

For the SANS experiments a HEPES buffer (10 mM HEPES, $100 \mathrm{mM} \mathrm{NaCl}$ ) in $\mathrm{D}_{2} \mathrm{O}$ was used, applying the concept of contrast variation (Knoll et al. 1981). Using heavy water the $\mathrm{pD}$ was adjusted to a value equivalent to $\mathrm{pH} 7.6$ using the conversion $\mathrm{pD}=0.929 \mathrm{pH}$ meter reading +0.42 (Lowe and Smith 1973). Ang II was added to the buffer to final concentrations of $0.1,0.5$ and $1 \mathrm{mM}$. As a control, a pure lipid sample was used.

Small-angle neutron scattering experiments were performed on the SANS 1 instrument at the FRG 1 research reactor at Helmholtz-Zentrum Geesthacht Centre for Materials and Coastal Research, Germany (Stuhrmann et al. 1995). The neutron wavelength was $8.5 \AA$, and the wavelength resolution was $10 \%$ (full width at half-maximum value). The range of scattering vectors $(0.004<q<$ $0.25 \AA^{-1}$ ) was obtained using four sample-to-detector distances from 0.7 to $9.7 \mathrm{~m}$. For the SANS measurements, the samples were equilibrated at various temperatures with a precision of $\pm 0.5^{\circ} \mathrm{C}$ in quartz cuvettes (Hellma, Muellheim, Germany) with a 1-mm path length. The raw SANS spectra were corrected for backgrounds from the solvent, sample cell and other sources by conventional procedures. The two-dimensional isotropic scattering spectra were averaged, normalized to the scattering of pure $\mathrm{H}_{2} \mathrm{O}$, and corrected for detector efficiency. Incoherent scattering of the buffer was subtracted.

\section{Vesicle preparation}

For the DLS and SANS experiments, unilamellar vesicles were used. DMPC respectively d54-DMPC was immersed in the different buffers to a final concentration of $5 \mathrm{mM}$ for DLS and $10 \mathrm{mM}$ for SANS, producing multilamellar vesicles (MLVs). The temperature of the dispersion was kept above $40^{\circ} \mathrm{C}$, and, after vortexing, unilamellar extruded vesicles were produced using an Avanti Mini Extruder (Avanti Polar Lipids, Alabaster, AL) and an Isopore polycarbonate filter (Millipore, Billerica, MA) of $0.1 \mu \mathrm{m}$ pore size. As extruded ULVs are generally contaminated with pauci-lamellar vesicles (PLVs), a small vesicle size 
was used to maximize the amount of ULVs (Kucerka et al. 2007). The MLVs were extruded an odd number of passes to avoid contamination of the sample by large and multilamellar vesicles, which might not have passed through the filter.

\section{SANS data analysis}

The analysis of the SANS data was performed using the software package described by Kline (2006) based on macros running under Igor Pro (WaveMetrics, Inc., Portland, OR). This data analysis software provides the possibilty to carry out non-linear data fitting, based on a variety of included form factors. To analyze our data in particular, the combination of form factors became necessary. Following the separated form factor approach as published by Kiselev et al. (2002), two form factors were summed up in order to reflect the changes in the sample with an appropriate model. One of the two form factors describes a lamellae, taking into account different scattering length densities for the chain and the head group region (Nallet et al. 1993; Javierre et al. 2000). Second, a form factor representing a polydisperse vesicle (Bartlett and Ottewill 1992) was used. The initially used scattering length densities (SLDs) were calculated values. The scattering length values for $\mathrm{D}_{2} \mathrm{O}$ were fixed. For the bilayer thickness, the length of the head group and the hydrophobic region of pure DMPC literature values were used as an orientation (Nagle and Tristram-Nagle 2000; Kucerka et al. 2008; Kiselev et al. 2006).

\section{Form factor of polydisperse vesicles}

The form factor (FF) for a vesicle with a polydisperse core with constant shell thickness was used as described by Bartlett and Ottewill (1992). To derive the relation and to define the parameters of the FF, a suspension of noninteracting particles was considered showing only coherent scattering and no multiple scattering.

$\frac{d \sum_{d \omega}}{d \mathbf{q}}=n\left\langle\left|\sum_{i(v)} b_{i} \exp \left(i \mathbf{q} \cdot \mathbf{r}_{i}\right)\right|^{2}\right\rangle$

$\mathrm{b}_{i}$ is the atomic scattering length density, $n$ is the number density of particles, and the sum is added over all atoms in the particle volume $v$. As SANS experiments do not yield information on the atomic scale, the atomic scattering lengths $b_{i}$ could be replaced by the locally averaged scattering length density defined as

$\rho(\mathbf{r})=\sum_{i} b_{i} \delta\left(\mathbf{r}-\mathbf{r}_{i}\right)$

$b_{i}$ is the scattering length of the atom at the position $r_{i}$. Replacing the sum in Eq. 3 by an integral yields an expression for the scattering from a suspension of noninteracting spheres

$\frac{d \Sigma}{d \omega}(\mathbf{q})=n F(\mathbf{q})^{2}$

where the scattering amplitude if defined by the Fourier transform

$F(\mathbf{q})=\int\left[\rho(\mathbf{r})-\rho_{m}\right] \exp (i \mathbf{q} \cdot \mathbf{r}) d \mathbf{r}$

$\rho_{m}$ is the scattering length density of the buffer. Assuming a spherically symmetric profile $\rho(r)$, the scattering amplitude for a single particle reduced to

$F(q)=4 \pi \int r^{2}\left[\rho(r)-\rho_{m}\right] \frac{\sin q r}{q r} d r$

The scattering profile has the form

$\rho(r)=\left\{\begin{array}{cc}\rho_{c} & r \leq r_{c} \\ \rho_{s} & r_{c}<r \leq r_{c}+\Delta\end{array}\right.$

with $r_{c}$ the radius of the spherical core and the scattering length density $\rho_{c}$ surrounded by a shell of the thickness $\Delta$ with the scattering length density $\rho_{s}$. Correspondingly, the particle scattering amplitude could be written as

$F(x)=\frac{4 \pi}{q^{3}}\left(\rho_{s}-\rho_{c}\right)\{\gamma j(x+\delta x)-j(x)\}$

$\rho=\Delta / r_{c}$ and $x=q r_{c}$. The function $j(x)=\sin x-x \cos x$. The scaled medium contrast $\gamma=\left(\rho_{m}-\rho_{s}\right) /\left(\rho_{c}-\rho_{s}\right)$ determines the relative proportion of the scattering from the core or the shell. To take into account the polydispersity of the vesicle, a particle distribution was introduced. For this, Eq. 5 needed to be averaged over the particle size distribution, assuming that only the size of the particle core varied, whereas the shell thickness $\Delta$ was constant. For this the single particle FF $F^{2}\left(q r_{c}\right)$ was replaced by the size average

$\bar{F}^{2}\left(q \bar{r}_{c}\right)=\int_{0}^{\infty} G\left(r_{c}\right) F^{2}\left(q r_{c}\right) d r_{c}$

$G\left(r_{c}\right)$ is the normalized probability to find a particle with a core radius between $r_{c}$, and $r_{c}+d r_{c}, \bar{r}_{c}$ is the mean core radius. For the distribution of the mean core radius, a Schulz distribution was picked. The normalized form of this distribution is

$G\left(r_{c}\right)=\frac{r_{c}^{Z}}{\Gamma(Z+1)}\left(\frac{Z+1}{\bar{r}_{c}}\right)^{Z+1} \cdot \exp \left[-\frac{r_{c}}{\overline{\bar{r}}_{c}}(Z+1)\right]$

in which $\bar{r}_{c}$ is the mean core radius, and $\mathrm{Z}$ is related to the normalized polydispersity $\sigma_{c}$ of the particle core radius distribution

$\sigma_{c}^{2}=\left(\frac{\bar{r}_{c}^{2}}{\bar{r}_{c}^{2}}-1\right)=\frac{1}{Z+1}$ 


\section{Lamellar form factor}

For the description of the bilayer, a lamellar FF was used as published by Nallet et al. (1993) and Javierre et al. (2000). To describe the different segments of the bilayer, a twosquare profile was defined, with two variable parameters for each segment. The segments were the head group with the thickness $\delta_{H}$ and the scattering length density $\sigma_{H}$, and accordingly the tail with the thickness $\delta_{T}$ and the scattering length density $\sigma_{T}$. To derive the form factor the following assumptions were made

$\Delta \sigma_{H}=\sigma_{H}-\sigma_{\text {solvent }}$

$\Delta \sigma_{T}=\sigma_{T}-\sigma_{H}$

$2\left(\delta_{H}+\delta_{T}\right)=$ total bilayer thickness

The resulting FF had the following format.

$$
\begin{aligned}
P(q)= & \frac{4}{q^{2}}\left\{\Delta \rho_{H}\left[\sin \left[q\left(\rho_{H}+\rho_{T}\right)\right]-\sin \left(q \rho_{T}\right)\right]\right. \\
& \left.+\Delta \rho_{T} \sin \left(q \delta_{T}\right)\right\}^{2}
\end{aligned}
$$

\section{Results}

Differential scanning calorimetry

In the DSC measurements we investigated pure DMPC samples and the same concentration of DMPC with different concentrations of Ang II. The data were normalized by the lipid concentration, and the baseline was subtracted. In Table 1 the thermodynamic data resulting from the DSC scans are listed, and in Fig. 1 the corresponding thermotropic traces are shown. The table comprises the values for the peak temperatures of the pre- and main transition. In addition, the enthalpy was calculated by integration of the area under the main transition peak and the pre-transition peak. Another indicator for changes in the phase behavior is the upper phase boundary, representing the temperature where half of the lipids are in the gel state and the other half in the fluid state (Feigenson 2007).
The results for pure DMPC dispersions were in good agreement with those of previous studies (Heimburg 2000). For pure DMPC a sharp main transition peak just below $24^{\circ} \mathrm{C}$ and in addition a pre-transition around $14^{\circ} \mathrm{C}$ were expected. From our measurement, a temperature of $23.6^{\circ} \mathrm{C}$ for the main transition and $13.8^{\circ} \mathrm{C}$ for the pre-transition resulted. Accordingly, the enthalpy for the main transition was calculated as 6.55 and as $1.37 \mathrm{~kJ} / \mathrm{mol}$ for the pretransition.

With increasing concentrations of Ang II, the peak temperature shifted towards higher temperatures, and the enthalpy increased. For the main transition, the enthalpy increased constantly with increasing concentrations of the peptide. The initial addition of the peptide led to an increase in enthalpy of about $1.5 \mathrm{~kJ} / \mathrm{mol}$. At higher concentrations, a smaller increase in the enthalpy of the main transition could be observed. For ever higher ratios, the enthalpy increased by about $600 \mathrm{~J} / \mathrm{mol}$.

In addition, the enthalpy of the pre-transition changed upon addition of the peptide. At the highest peptide:lipid ratio (1:100), an increase of $72 \mathrm{~J} / \mathrm{mol}$ was calculated. For the peptide lipid ratio of 1:50, the enthalpy increased more than $200 \mathrm{~J} / \mathrm{mol}$. Finally, for the peptide:lipid ratio of 1:10, the enthalpy increased again by about $200 \mathrm{~J} / \mathrm{mol}$ compared to the previous ratio. For the highest concentration of the peptide and a ratio of peptide to lipid of 1:5, the enthalpy of the pre-transition dropped slightly compared to the next lower peptide concentration.

Studying the temperature change induced by the peptide, again the temperature of the main transition and of the pre-transition was compared for pure DMPC and samples containing Ang II in addition. The temperature of the main transition shifted between 0.4 and $0.6^{\circ} \mathrm{C}$ as a response to Ang II binding. Comparing the temperature of the pure DMPC sample and after addition of peptide with a peptide:lipid ratio of $1: 100$, an increase in the transition temperature of $0.4^{\circ} \mathrm{C}$ was observed. For the higher peptide concentrations, only a small further increase of the temperature could be seen. The analysis of the pre-transition temperature gave a similar result. The temperature

Table 1 Enthalpy and peak temperature of the main transition and pre-transition, as well as the upper phase boundary for pure DMPC

\begin{tabular}{|c|c|c|c|c|c|}
\hline Sample & DMPC & $1: 100$ & $1: 50$ & $1: 10$ & $1: 5$ \\
\hline $\begin{array}{l}\text { Enthalpy main transition } \\
C_{\mathrm{p}}(\mathrm{J} / \mathrm{mol} \operatorname{deg})\end{array}$ & $6,558.6 \pm 918.2$ & $8,040.47 \pm 1,225.66$ & $8,818.38 \pm 1,234.57$ & $9,602.9 \pm 1,344.4$ & $10,361.4 \pm 1,450.59$ \\
\hline Pre-transition $C_{\mathrm{p}}(\mathrm{J} / \mathrm{mol} \mathrm{deg})$ & $1,374.09 \pm 192.37$ & $1,446.95 \pm 202.573$ & $1,645 \pm 230.3$ & $1,856.25 \pm 259.87$ & $1,765.54 \pm 247.17$ \\
\hline $\begin{array}{l}\text { Peak temperature main } \\
\text { temperature }\left({ }^{\circ} \mathrm{C}\right)\end{array}$ & 23.59 & 23.99 & 24.01 & 24.04 & 24.03 \\
\hline $\begin{array}{l}\text { Peak temperature } \\
\text { pre-temperature }\left({ }^{\circ} \mathrm{C}\right)\end{array}$ & 13.84 & 15.19 & 15.37 & 15.12 & 15.05 \\
\hline Upper phase boundary $\left({ }^{\circ} \mathrm{C}\right)$ & 23.64 & 24.07 & 24.07 & 24.11 & 24.16 \\
\hline
\end{tabular}
dispersions and DMPC mixed with Ang II in the peptide:lipid ratios 1:100, 1:50, 1:10 and 1:5 

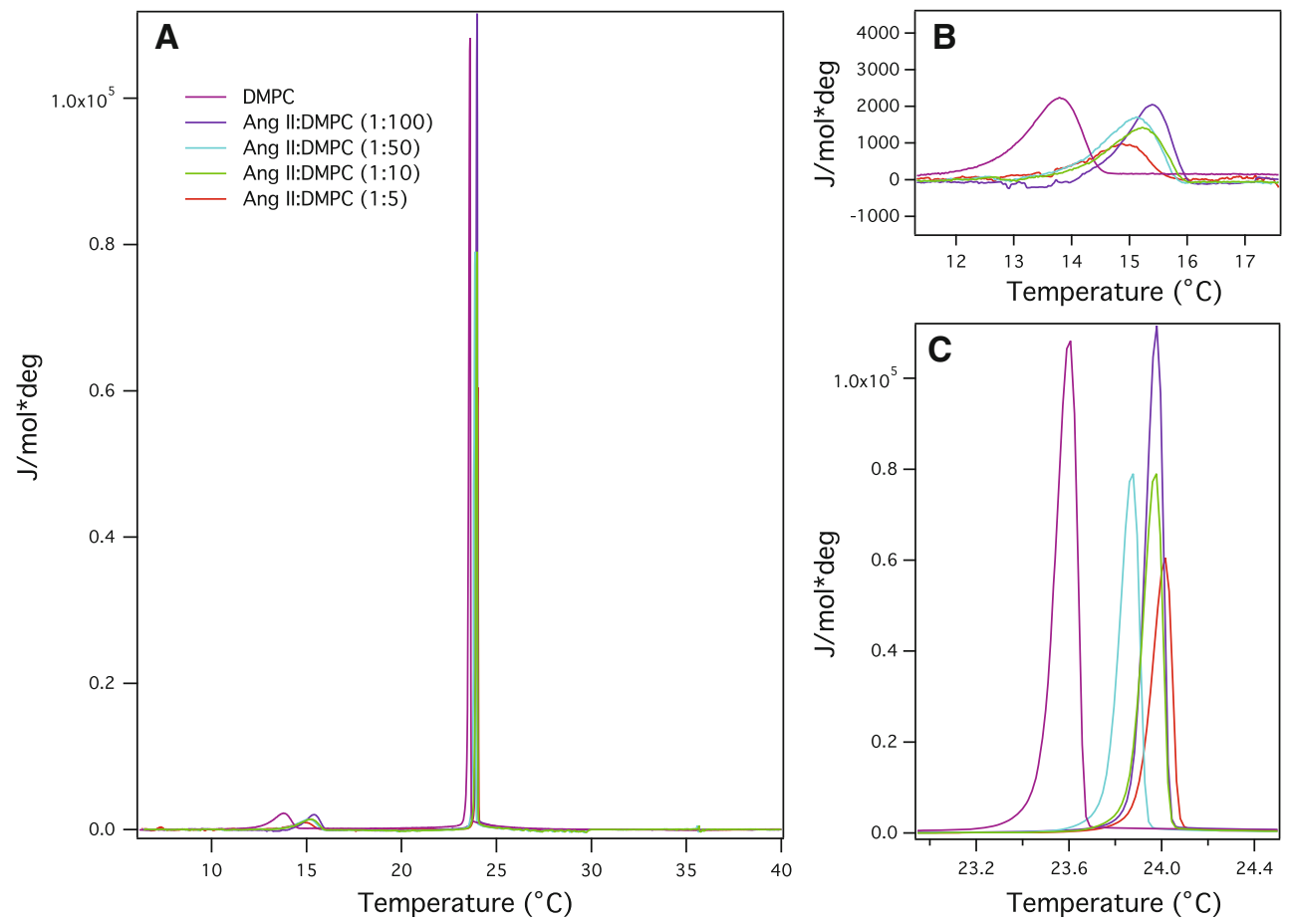

Fig. 1 Excess heat capacity curves of DMPC and mixtures of peptide and lipid with ratios of 1:100, 1:50, 1:10 and 1:5. a Displays the whole thermogram. b Shows a magnification of the pre-transition region and $\mathbf{c}$ a magnification of the main transition peaks

increased by $1.3^{\circ} \mathrm{C}$ for the addition of the peptide at the lowest concentration and by around $0.2^{\circ} \mathrm{C}$ for the next higher peptide concentration. For the higher peptide concentrations, the result was different. The temperature always stayed above $15^{\circ} \mathrm{C}$. Another indicator for changes occurring at the membrane is the upper phase boundary, the temperature where an equal number of lipids are in the fluid state and in the gel state. For DMPC the upper phase boundary was calculated as $23.64^{\circ} \mathrm{C}$, and it increased by $0.42^{\circ} \mathrm{C}$ for the two lowest peptide concentrations. For the two higher concentrations the temperature increased by $0.46^{\circ} \mathrm{C}$.

As the pre-transition temperature is more sensitive to changes at the membrane surface, a change in the pretransition temperature points to an interaction of the peptide with the membrane surface. Upon molecules interacting not only with the surface, but also at the interface region or in the hydrophobic region, the pretransiton will vanish completely. For the present study, the pretransition remained visible in the data and was shifted towards higher temperatures by more than $1^{\circ} \mathrm{C}$. The peak broadened, and the enthalpy increased.

The enthalpy of the main transition also increased except at the highest peptide concentration. This non-linear increase in enthalpy supported a weak binding and interaction with the surface of the bilayer. With a decreasing peptide:lipid ratio, a saturation level was reached where no further peptide molecules bound to the surface. We will further elaborate on this fact after introducing the results from the scattering experiments. The results from the DSC gave a first insight into the interaction of the membrane with the peptide, and it can be concluded that the interaction was weak and mainly took place at the surface of the membrane. Keeping in mind that calorimetric studies only trace changes, having an impact on the enthalpy of the system, not all structural changes could be recorded. To get a more detailed view of the structural changes, small angle scattering was applied.

Dynamic light scattering

Dynamic light scattering was used to gain a first insight into the structural changes occurring in the vesicles upon addition of the peptide; the results are shown in Fig. 2 and Table 2. The main information gained from light scattering experiments is the hydrodynamic radius. For the experiments extruded vesicles were used, and the buffer contained the peptide in different concentrations. As a main result we could show that the hydrodynamic radius decreases upon addition of the peptide. Initially, a hydrodynamic radius of about $85 \mathrm{~nm}$ was measured for DMPC above the phase transition. Lowering the temperature led to a gradual decrease in the hydrodynamic radius. Below the phase transition the radius reached around $65 \mathrm{~nm}$. The difference in the radius can be explained by the melting of the lipid acyl chains, increasing the area per lipid by about $30 \%$. 


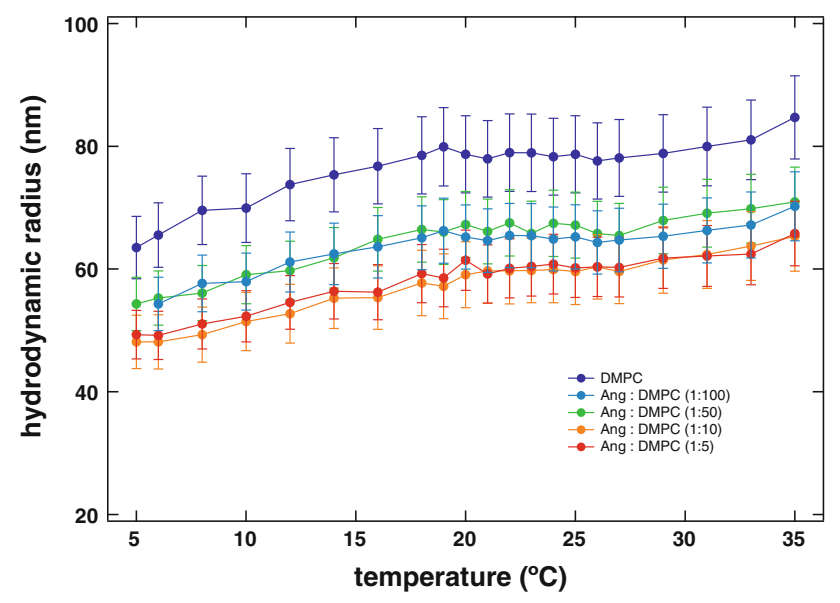

Fig. 2 Dynamic light scattering data showing the hydrodynamic radii calculated for temperatures between 5 and $35^{\circ} \mathrm{C}$. The bars indicate the error resulting from the polydispersity of the vesicles. The line between the data points is added to provide better visibility

\section{Small angle neutron scattering}

To gain an insight into the structural changes occurring on the membrane level, small angle neutron scattering (SANS) was applied. Diluted dispersions, containing about $1 \mathrm{w} \%$ lipid, were used as sample material to ensure that only single particle form factors (FFs) were measured. The dispersion was extruded to avoid aggregation and periodic packing of bilayers that could have given rise to Bragg peaks. All samples were measured with respect to the different lipid phases at 15,22 and $35^{\circ} \mathrm{C}$.

After general corrections, averaging and integration of the data, detailed data analysis was performed using the separated form factor (SFF) approach (Kiselev et al. 2002). Two FFs were combined, leading to the possibility to describe the vesicle and the structure of the lamellae at the same time. In detail, the first FF described the tail length and the thickness of the head group and the corresponding scattering length densities (SLDs), respectively. The second FF was used to describe the overall structure of the vesicle, taking into account the vesicle size, the bilayer thickness and the polydispersity of the vesicle. For all experiments, chain deuterated DMPC (d54-DMPC) was used, having the advantage that a large SLD contrast difference exists between the head group and the acyl chains. A penetration of the peptide into the hydrophobic core would become visible instantly in the data. The results are displayed in Fig. 3 and in Table 3 for the different temperatures.

At first the data from the pure d54-DMPC samples were fitted, starting with the data set where the lipids were in the fluid state as only limited literature is available for the gel respectively ripple phase. The values for d54-DMPC in the fluid phase literature values were taken where possible or the values for DPPC were used taking into account the
Table 2 Hydrodynamic radii from dynamic light scattering experiments for pure DMPC dispersions and lipid peptide mixtures with different ratios shown for temperatures between 35 and $5^{\circ} \mathrm{C}$

\begin{tabular}{llllll}
\hline $\begin{array}{l}\text { Temperature } \\
\left({ }^{\circ} \mathrm{C}\right)\end{array}$ & \multicolumn{4}{l}{ Hydrodynamic radius $(\mathrm{nm})$} \\
\cline { 2 - 6 } & DMPC & $(1: 100)$ & $(1.50)$ & $(1: 10)$ & $(1: 5)$ \\
\hline 35 & $84.72 \pm 6.78$ & $70.22 \pm 5.62$ & $70.93 \pm 5.67$ & $65.31 \pm 5.22$ & $65.78 \pm 5.26$ \\
33 & $81.04 \pm 6.48$ & $67.17 \pm 5.37$ & $69.84 \pm 5.59$ & $63.75 \pm 5.10$ & $62.45 \pm 5.00$ \\
31 & $79.98 \pm 6.40$ & $66.30 \pm 5.30$ & $69.10 \pm 5.53$ & $62.37 \pm 4.99$ & $62.13 \pm 4.97$ \\
29 & $78.84 \pm 6.31$ & $65.35 \pm 5.23$ & $67.91 \pm 5.43$ & $61.48 \pm 4.92$ & $61.78 \pm 4.94$ \\
27 & $78.10 \pm 6.25$ & $64.74 \pm 5.18$ & $65.46 \pm 5.24$ & $59.60 \pm 4.77$ & $60.25 \pm 4.82$ \\
26 & $77.61 \pm 6.21$ & $64.33 \pm 5.15$ & $65.79 \pm 5.26$ & $60.35 \pm 4.83$ & $60.36 \pm 4.83$ \\
25 & $78.70 \pm 6.30$ & $65.24 \pm 5.22$ & $67.15 \pm 5.37$ & $59.60 \pm 4.77$ & $60.17 \pm 4.81$ \\
24 & $78.30 \pm 6.26$ & $64.91 \pm 5.19$ & $67.44 \pm 5.40$ & $59.92 \pm 4.79$ & $60.80 \pm 4.86$ \\
23 & $78.93 \pm 6.31$ & $65.43 \pm 5.23$ & $65.80 \pm 5.26$ & $59.77 \pm 4.78$ & $60.42 \pm 4.83$ \\
22 & $78.97 \pm 6.32$ & $65.46 \pm 5.24$ & $67.52 \pm 5.40$ & $59.71 \pm 4.78$ & $60.10 \pm 4.81$ \\
21 & $77.96 \pm 6.24$ & $64.62 \pm 5.17$ & $66.12 \pm 5.29$ & $59.69 \pm 4.78$ & $59.21 \pm 4.74$ \\
20 & $78.69 \pm 6.29$ & $65.22 \pm 5.22$ & $67.26 \pm 5.38$ & $59.08 \pm 4.73$ & $61.42 \pm 4.91$ \\
19 & $79.91 \pm 6.39$ & $66.24 \pm 5.30$ & $66.03 \pm 5.28$ & $57.18 \pm 4.57$ & $58.54 \pm 4.68$ \\
18 & $78.52 \pm 6.28$ & $65.09 \pm 5.21$ & $66.46 \pm 5.32$ & $57.72 \pm 4.62$ & $59.25 \pm 4.74$ \\
16 & $76.75 \pm 6.14$ & $63.62 \pm 5.09$ & $64.84 \pm 5.19$ & $55.34 \pm 4.43$ & $56.24 \pm 4.50$ \\
14 & $75.35 \pm 6.03$ & $62.46 \pm 5.00$ & $61.83 \pm 4.95$ & $55.25 \pm 4.42$ & $56.39 \pm 4.51$ \\
12 & $73.75 \pm 5.90$ & $61.13 \pm 4.89$ & $59.75 \pm 4.78$ & $52.71 \pm 4.22$ & $54.56 \pm 4.36$ \\
10 & $69.92 \pm 5.59$ & $57.96 \pm 4.64$ & $59.07 \pm 4.73$ & $51.45 \pm 4.12$ & $52.30 \pm 4.18$ \\
8 & $69.57 \pm 5.57$ & $57.66 \pm 4.61$ & $56.08 \pm 4.49$ & $49.31 \pm 3.95$ & $51.06 \pm 4.08$ \\
6 & $65.53 \pm 5.24$ & $54.32 \pm 4.35$ & $55.27 \pm 4.42$ & $48.14 \pm 3.85$ & $49.20 \pm 3.94$ \\
5 & $63.50 \pm 5.08$ & & $54.33 \pm 4.35$ & $48.13 \pm 3.85$ & $49.32 \pm 3.95$ \\
\hline & & & &
\end{tabular}




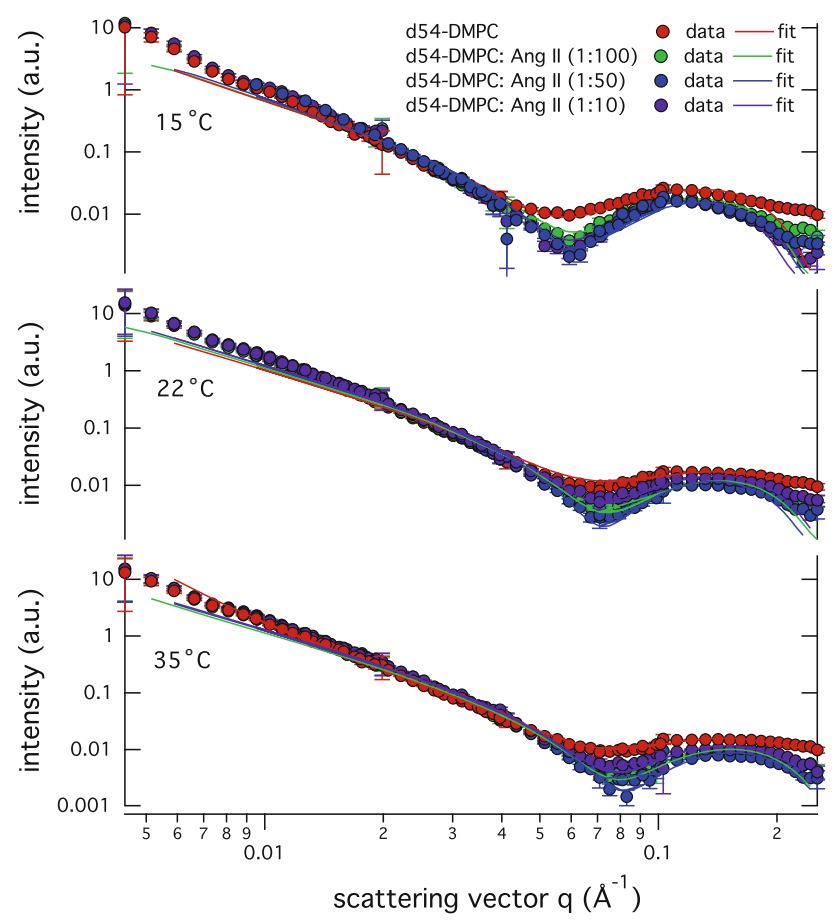

Fig. 3 Small angle scattering data and according fits. Data are shown for experiments on pure d54-DMPC and mixtures of peptide and lipid of 1:100, 1:50, 1:10 and 1:5. The lines indicate the fits performed for the individual data sets

difference in tail length (Nagle and Tristram-Nagle 2000; Kucerka et al. 2008; Kiselev et al. 2006). Based on this initial data, a fit to the experimental data became possible. For d54-DMPC in the fluid phase, the tail length was fitted to $13.8 \AA \pm 1.65 \AA$ with a SLD of $7.1 \cdot 10^{-6} \AA^{-2}$ and for the head group a thickness of $5.47 \pm 0.65 \AA$ with a SLD of $1.83 \cdot 10^{-6} \AA^{-2} \pm 0.21 \cdot 10^{-6} \AA^{-2}$. This sums up to a membrane thickness of $38.54 \AA$, which is in good agreement with the literature values (Kucerka et al. 2008; Kiselev et al. 2006). With these parameters the according fits for the d54-DMPC data sets at lower temperatures were performed. For the sample at $15^{\circ} \mathrm{C}$, a smaller thickness of the head group and a longer hydrophobic region was expected. The head group was slightly tilted in the ripple phase, and therefore the head group appeared smaller in the ripple phase. Unmolten acyl chains are about $20 \%$ longer than the molten ones, making a difference of about $3 \AA$ between the two states. For the sample at $22^{\circ} \mathrm{C}$, the values should lie in between. The resulting thickness of the head group at $15^{\circ} \mathrm{C}$ was $4 \AA$ and $4.74 \AA$ at $22^{\circ} \mathrm{C}$, ; correspondingly the tail length at $15^{\circ} \mathrm{C}$ was fitted with 16 . $\AA$ and for $22^{\circ} \mathrm{C}$ with $14.5 \AA$. Based on the fits for the pure lipid samples, the data with increasing peptide:lipid ratios were fitted. At $15^{\circ} \mathrm{C}$ the acyl chains were fitted with a constant length of $16.3 \AA \pm 0.1 .956 \AA$. The thickness of the head group increased with the amount of peptide added from $4 \AA \pm 0.48 \AA$ for pure d54-DMPC to $4.59 \AA \pm 0.55 \AA$ at a peptide:lipid ratio of $1: 100,5.48 \AA \pm 0.657 \AA$ at a peptide:lipid ratio of $1: 50$ and finally to $7.76 \AA \pm 0.93 \AA$ at a ratio of $1: 10$. The fits for the sample incubated at $22^{\circ} \mathrm{C}$ resulted in an slight decrease of the tail length, going from $14.5 \AA \pm 1.74 \AA, 14.85 \AA \pm 1.782 \AA$ to $13.6 \AA \pm 1.63 \AA$ with increasing peptide concentration. In turn the head group size increased from $4.64 \AA \pm 0.808 \AA$ over $6.12 \AA \pm$ $0.734 \AA$ and $7.67 \AA \pm 0.92 \AA$ to $7.4 \AA \pm 0.88 \AA$ for the highest peptide amount in the sample. In the fluid phase, at $35^{\circ} \mathrm{C}$, the tail length again decreased slightly from 13.53 $\AA \pm 1.623 \AA$ (peptide:lipid ratio 1:100) to $13.4 \AA \pm 1.6 \AA$ (1:50) down to $13 \AA \pm 1.5 \AA$ (1:10). The fitted values for the head group were $6 \AA \pm 0.7 \AA, 6.65 \AA \pm 0.798 \AA$ and $8.6 \AA \pm 1.03 \AA$ with increasing peptide:lipid ratio.

In addition to changes in the length of the head group and the hydrophobic tail, the SLDs were adjusted during the fitting procedure. At $15^{\circ} \mathrm{C}$ the SLD for the head group changed from $1.57 \cdot 10^{-6} \AA^{-2} \pm 0.188 \cdot 10^{-6} \AA^{-2}$ over $1.9 \cdot 10^{-6} \AA^{-2} \pm 0.22 \cdot 10^{-6} \AA^{-2}$ to $3.3 \cdot 10^{-6} \AA^{-2} \pm$ $0.39 \cdot 10^{-6} \AA^{-2}$ and accordingly for the acyl chains dropped slightly to $7.1 \cdot 10^{-6} \AA^{-2}$. At $22^{\circ} \mathrm{C}$ the SLD of the head group also increased with increasing amounts of peptides by around $2 \cdot 10^{-6} \AA^{-2}$, whereas the SLD of the tails stayed constant. Finally, for $35^{\circ} \mathrm{C}$ the SLD of the head group stayed constant for the low peptide concentrations, but increased to $3.14 \cdot 10^{-6} \AA^{-2}$ for a peptide:lipid ratio of $1: 10$. The SLD of the tail decreased only insignificantly.

\section{Discussion}

From the results of the DSC and the SANS data, a concentration-dependent model for the binding of Ang II to membranes can be suggested. The DSC experiments resulted in a shift of the main transition towards higher temperatures; hence, a preferential binding to the gel phase was indicated. This was supported by the SANS data, as the increase in head group thickness at $15^{\circ} \mathrm{C}$ was the highest one for all the studied temperatures, accompanied by the highest increase in the SLD of the head group. The thickness of the tail stayed unaffected, which can be explained by the close packing of the acyl chains in the gel phase, which could not be accessed by the peptide, even at high peptide concentrations.

At $35^{\circ} \mathrm{C}$ the head group thickness also increased upon the binding of the peptide, and in addition the tail length was slightly decreased, pointing to a penetration of the peptide into the interface of the head group and the acyl 
Table 3 Small angle neutron scattering data fitting parameter

\begin{tabular}{|c|c|c|c|c|c|}
\hline Temp $\left({ }^{\circ} \mathrm{C}\right)$ & Sample & d54-DMPC & $1: 100$ & $1: 50$ & $1: 10$ \\
\hline \multirow[t]{9}{*}{15} & Tail length $(\AA)$ & $16 \pm 1.9$ & $16.3 \pm 1.95$ & $16.3 \pm 1.95$ & $16.3 \pm 1.95$ \\
\hline & Head group thickness $(\AA)$ & $4 \pm 0.4$ & $4.59 \pm 0.55$ & $5.48 \pm 0.657$ & $7.76 \pm 0.931$ \\
\hline & SLD tails $\left(\cdot 10^{-6} \AA^{-2}\right)$ & $7.2 \pm 0.85$ & $7.2 \pm 0.86$ & $7.15 \pm 0.85$ & $7.1 \pm 0.85$ \\
\hline & SLD head group $\left(\cdot 10^{-6} \AA^{-2}\right)$ & $2.04 \pm 0.244$ & $1.57 \pm 0.188$ & $1.9 \pm 0.22$ & $3.3 \pm 0.39$ \\
\hline & Background $\left(\mathrm{cm}^{-1}\right)$ & $0.001 \pm 0.0001$ & $0.001 \pm 0.0001$ & $0.001 \pm 0.0001$ & $0.001 \pm 0.0001$ \\
\hline & Average core radius $(\AA)$ & $760.5 \pm 91.2$ & $630.4 \pm 75.6$ & $633.3 \pm 75.9$ & $552.9 \pm 66.3$ \\
\hline & Overall polydispersity & $0.28 \pm 0.033$ & $0.28 \pm 0.033$ & $0.28 \pm 0.033$ & $0.28 \pm 0.033$ \\
\hline & SLD core and solvent $\left(\cdot 10^{-6} \AA^{-2}\right)$ & $6.3 \pm 0.756$ & $6.36 \pm 0.763$ & $6.36 \pm 0.763$ & $6.36 \pm 0.763$ \\
\hline & SLD shell $\left(\cdot 10^{-6} \AA^{-2}\right)$ & $4.78 \pm 0.573$ & $4.78 \pm 0.573$ & $4.78 \pm 0.573$ & $4.78 \pm 0.573$ \\
\hline \multirow[t]{9}{*}{22} & Tail length $(\AA)$ & $14.5 \pm 1.74$ & $14.5 \pm 1.74$ & $14.85 \pm 1.782$ & $13.6 \pm 1.632$ \\
\hline & Head group thickness $(\AA)$ & $4.74 \pm 0.808$ & $6.12 \pm 0.734$ & $7.67 \pm 0.92$ & $7.4 \pm 0.88$ \\
\hline & SLD tails $\left(\cdot 10^{-6} \AA^{-2}\right)$ & $7.2 \pm 0.86$ & $7.2 \pm 0.86$ & $7.1 \pm 0.85$ & $7.15 \pm 0.85$ \\
\hline & SLD head group $\left(\cdot 10^{-6} \AA^{-2}\right)$ & $2.09 \pm 0.25$ & $1.95 \pm 0.234$ & $1.65 \pm 0.198$ & $3.56 \pm 0.427$ \\
\hline & Background $\left(\mathrm{cm}^{-1}\right)$ & $0.001 \pm 0.0001$ & $0.001 \pm 0.0001$ & $0.001 \pm 0.0001$ & $0.001 \pm 0.0001$ \\
\hline & Average core radius $(\AA)$ & $789 \pm 94.6$ & $650.8 \pm 78.09$ & $675.2 \pm 81.02$ & $597 \pm 71.6$ \\
\hline & Overall polydispersity & $0.28 \pm 0.033$ & $0.28 \pm 0.033$ & $0.28 \pm 0.033$ & $0.28 \pm 0.033$ \\
\hline & SLD core and solvent $\left(\cdot 10^{-6} \AA^{-2}\right)$ & $6.36 \pm 0.763$ & $6.36 \pm 0.763$ & $6.36 \pm 0.763$ & $6.36 \pm 0.763$ \\
\hline & SLD shell $\left(\cdot 10^{-6} \AA^{-2}\right)$ & $4.78 \pm 0.573$ & $4.78 \pm 0.573$ & $4.78 \pm 0.573$ & $4.78 \pm 0.573$ \\
\hline \multirow[t]{9}{*}{35} & Tail length $(\AA ̊)$ & $13.8 \pm 1.65$ & $13.53 \pm 1.62$ & $13.4 \pm 1.6$ & $13 \pm 1.56$ \\
\hline & Head group thickness $(\AA)$ & $5.47 \pm 0.656$ & $6 \pm 0.7$ & $6.65 \pm 0.798$ & $8.6 \pm 1.03$ \\
\hline & SLD tails $\left(\cdot 10^{-6} \AA^{-2}\right)$ & $7.1 \pm 0.85$ & $7.1 \pm 0.86$ & $7.1 \pm 0.85$ & $7 \pm 0.84$ \\
\hline & SLD head group $\left(\cdot 10^{-6} \AA^{-2}\right)$ & $1.83 \pm 0.219$ & $1.79 \pm 0.214$ & $1.5 \pm 0.18$ & $3.14 \pm 0.376$ \\
\hline & Background $\left(\mathrm{cm}^{-1}\right)$ & $0.001 \pm 0.0001$ & $0.001 \pm 0.0001$ & $0.001 \pm 0.0001$ & $0.001 \pm 0.0001$ \\
\hline & Average core radius $(\AA)$ & $847 \pm 101.6$ & $702.2 \pm 84.26$ & $709 \pm 85.08$ & $653 \pm 78.36$ \\
\hline & Overall polydispersity & $0.28 \pm 0.033$ & $0.28 \pm 0.033$ & $0.28 \pm 0.033$ & $0.28 \pm 0.033$ \\
\hline & SLD core and solvent $\left(\cdot 10^{-6} \AA^{-2}\right)$ & $6.36 \pm 0.763$ & $6.36 \pm 0.763$ & $6.36 \pm 0.763$ & $6.36 \pm 0.763$ \\
\hline & SLD shell $\left(\cdot 10^{-6} \AA^{-2}\right)$ & $4.78 \pm 0.573$ & $4.78 \pm 0.573$ & $4.78 \pm 0.573$ & $4.78 \pm 0.573$ \\
\hline
\end{tabular}

chains. Close to the phase transition at $22^{\circ} \mathrm{C}$, this effect was even more pronounced, as the membrane was most flexible in this temperature region. This tendency was supported by the corresponding changes in the SLDs. At all temperatures the SLD of the tails stayed basically constant, whereas it increased for the head group region. This increase in the SLD was caused by the interaction of the peptide. The SLD of the peptide was lower than that of the lipid head group, but the penetration of $\mathrm{D}_{2} \mathrm{O}$ molecules into this region caused an increase in the SLD. For the samples at $22^{\circ} \mathrm{C}$, the SLD of the tails again stayed constant, and the one for the head group increased. At $35^{\circ} \mathrm{C}$ the SLD of the tails again could be considered as constant, and the SLD of the head only changed for high concentrations of peptides.

Our data give clear evidence for the adsorption of the peptide to the membrane surface. A tendency for preferred binding at the gel phase was evident, and only at high concentrations did the peptide start to penetrate closer to the interface between the head group and the acyl chains.

Looking at the previously published studies, it becomes evident that they focus on the structure of the peptide in different chemical environments. Various experiments aimed to elucidate the peptide structure in the receptor, which would lead to a basis for the rational design of Ang II peptidomimetics with potent antagonist properties. So far, no experimental structural information about the peptide bound to the $\mathrm{AT}_{1}$ receptor is available, and therefore a number of secondary structural features under various conditions in attempts to mimic the natural environment of the receptor have been analyzed (Surewicz and Mantsch 1988; Garcia et al. 1992; Shin and Yoo 1996; Cho and Asher 1996; Joseph et al. 1995), and in vitro binding studies of Losartan or Ang II have been made (Chansel et al. 1994). 
Fatigati and Peach postulated a lipid milieu as the local environment for Ang II; therefore, our interest focused on the concentration-dependent interaction of Ang II on model membranes and the structural changes induced by the insertion, studied at different temperatures (Fatigati and Peach 1988).

For the $\mathrm{AT}_{1}$ receptor antagonist Losartan binding is facilitated by binding of the antagonist to the membrane surface and diffusion through the membrane bilayer prior to binding of the receptor (Zoumpoulakis et al. 2003). Different from the mechanism proposed for Losartan, the adsorption of Ang II to the membrane surface may be involved in the translocation of the receptor. The binding of Ang II to the membrane can facilitate the transfer of the peptide to the active pocket in the receptor. Along with this discussion, our study provides new insight into the interaction of Ang II with model membranes, in this case DMPC vesicles. It can be concluded that Ang II influences the hydration of the head group and that this facilitates the insertion of the receptor in the membrane and subsequently the activation of the receptor by the peptide.

\section{Conclusion}

In conclusion, we could show that Ang II is in interaction with the surface of the membrane depending on the peptide concentration and the phase of the lipids. At low temperatures the peptide stayed on the surface and in the head group region. With increasing temperatures and increased fluctuations close to the temperature of the phase transition, a deeper penetration of the peptide into the bilayer is enabled. Ang II alters the hydration of the lipid head groups, facilitating possible refolding of the receptor in the membrane and the subsequent binding of Ang II in the active site.

In addition, we showed here that SANS is a suitable tool to study the interaction of a membrane-active peptide with the lipid surface. Key is the data analysis, as the combination of separated form factors gives rise to a variety of parameters that can describe a system on the level of the lamellae up to changes in the overall vesicle size. SANS and the method of contrast variation give rise to an increase in contrast, enabling a label-free sample preparation. The use of chain deuterated DMPC has the advantage that the contrast between the head group and the acyl chains is comparably high, such as the one between $\mathrm{D}_{2} \mathrm{O}$ and the head group.

Acknowledgments This research project was supported by the European Commission under the 6th Framework Programme through the Key Action: Strengthening the European Research Area, Research Infrastructures, contract no. RII3-CT-2003-505925. J.P. has been supported by the European Commission under the 6th Framework Programme through the Marie-Curie Action: BIOCONTROL, contract no. MCRTN 33439. The authors would like to thank the staff of the cSAXS group at the Paul Scherrer Institute for use of the light scattering equipment

\section{References}

Alves ID, Goasdoué N, Correia I, Aubry S, Galanth C, Sagan S, Lavielle S, Chassaing G (2008) Membrane interaction and perturbation mechanisms induced by two cationic cell penetrating peptides with distinct charge distribution. BBA-Gen Sub 1780(7-8):948-959

Balgavý P, Dubnicková M, Kucerka N, Kiselev MA, Yaradaikin SP, Uhríková D (2001) Bilayer thickness and lipid interface area in unilamellar extruded 1,2-diacylphosphatidylcholine liposomes: a small-angle neutron scattering study. Biochim Biophys Acta 1512(1):40-52

Bartlett P, Ottewill R (1992) A neutron-scattering study of the structural of a bimodal colloidal crystal. J Chem Phys 96(4):3306-3318

Bruylants G, Wouters J, Michaux C (2005) Differential scanning calorimetry in life science: thermodynamics, stability, molecular recognition and application in drug design. Curr Med Chem 12(17):2011-20

Burckbuchler V, Wintgens V, Leborgne C, Lecomte S, Leygue N, Scherman D, Kichler A, Amiel C (2008) Development and characterization of new cyclodextrin polymer-based dna delivery systems. Bioconjug Chem 19(12):2311-2320

Chansel D, Bizet T, Vandermeersch S, Pham P, Levy B, Ardaillou R (1994) Differential regulation of angiotensin ii and losartan binding sites in glomeruli and mesangial cells. Am J Physiol 266(3 Pt 2):F384-93

Chini B, Parenti M (2004) G-protein coupled receptors in lipid rafts and caveolae: how, when and why do they go there? J Mol Endocrinol 32:325-338

Cho NJ, Asher SA (1996) Uv resonance raman and absorption studies of angiotensin ii conformation in lipid environments. Biospectroscopy 2(2):71-82

Costerousse O, Jaspard E, Wei L, Corvol P, Alhenc-Gelas F (1992) The angiotensin i-converting enzyme (kininase ii): molecular organization and regulation of its expression in humans. J Cardiovasc Pharmacol 20(Suppl 9):S10-5

de Gasparo M, Catt KJ, Inagami T, Wright JW, Unger T (2000) International union of pharmacology. xxiii. the angiotensin ii receptors. Pharmacol Rev 52(3):415-472

Dézsi L (2000) Fibrinolytic actions of ace inhibitors: a significant plus beyond antihypertensive therapeutic effects. Cardiovasc Res 47(4):642-644

Dimsdale JE, Kolterman O, Koda J, Nelesen R (1996) Effect of race and hypertension on plasma amylin concentrations. Hypertension 27(6): 1273-1276

Fatigati V, Peach MJ (1988) Development of a new fluorescent angiotensin ii probe. Am J Physiol 255(4 Pt 1):C452-C458

Feigenson GW (2007) Phase boundaries and biological membranes. Annual Rev Biophys Biomol Struct 36:63-77

Gallová J, Uhríková D, Kucerka N, Teixeira J, Balgavý P (2008) Hydrophobic thickness, lipid surface area and polar region hydration in monounsaturated diacylphosphatidylcholine bilayers: Sans study of effects of cholesterol and beta-sitosterol in unilamellar vesicles. Biochim Biophys Acta 1778(11):2627-2632

Garcia KC, Ronco PM, Verroust PJ, Brünger AT, Amzel LM (1992) Three-dimensional structure of an angiotensin ii-fab complex at 3 a: hormone recognition by an anti-idiotypic antibody. Sci Agric 257(5069):502-507

Han M, Mei Y, Khant H, Ludtke Steven J (2009) Characterization of antibiotic peptide pores using cryo-em and comparison to neutron scattering. Biophys J 97(1):164-172 
Heimburg T (2000) A model for the lipid pretransition: coupling of ripple formation with the chain-melting transition. Biophys $\mathrm{J}$ 78(3):1154-1165

Hong F, Ming L, Yi S, Zhanxia L, Yongquan W, Chi L (2008) The antihypertensive effect of peptides: a novel alternative to drugs? Peptides 29(6):1062-1071

Huang HW (2000) Action of antimicrobial peptides: two-state model. Biochem Cell Biol 39(29):8347-52

Jacobson K, Mouritsen O, Anderson R (2007) Lipid rafts: at a crossroad between cell biology and physics. Nat Cell Biol 9(1):7-14. doi:10.1038/ncb0107-7

Javierre I, Nallet F, Bellocq AM, Maugey M (2000) Structure and dynamic properties of a polymer-induced sponge phase. J Phys Condens Matter 12:A295-A299

Joseph MP, Maigret B, Bonnafous JC, Marie J, Scheraga HA (1995) A computer modeling postulated mechanism for angiotensin ii receptor activation. J Protein Chem 14(5):381-98

Jung D, Powers JP, Straus SK, Hancock REW (2008) Lipid-specific binding of the calcium-dependent antibiotic daptomycin leads to changes in lipid polymorphism of model membranes. Chem Phys Lipids 154(2):120-128

Kiselev M, Lesieur P, Kisselev AM, Lombardo D, Aksenov V (2002) Model of separated form factors for unilamellar vesicles. Appl Phys A Mater Sci Process 74(Suppl):S1654-S1656

Kiselev MA, Zemlyanaya EV, Aswal VK, Neubert RHH (2006) What can we learn about the lipid vesicle structure from the small-angle neutron scattering experiment? Eur Biophys J 35(6):477-493

Kline SR (2006) Reduction and analysis of sans and usans data using igor pro. J Appl Crystallogr 39(6):895-900

Knoll W, Haas J, Stuhrmann HB, Fuldner HH, Vogel H, Sackmann E (1981) Small-angle neutron scattering of aqueous dispersions of lipids and lipid mixtures. a contrast variation study. J Appl Crystallogr 14(3):191-202

Knoll W, Ibel K, Sackmann E (1981) Small-angle neutron scattering study of lipid phase diagrams by the contrast variation method. Biochemisitry 20(22):6379-6383

Koukoulitsa C, Kyrikou I, Demetzos C, Mavromoustakos T (2006) The role of the anticancer drug vinorelbine in lipid bilayers using differential scanning calorimetry and molecular modeling. Chem Phys Lipids 144(1):85-95

Kucerka N, Nagle JF, Sachs JN, Feller SE, Pencer J, Jackson A, Katsaras J (2008) Lipid bilayer structure determined by the simultaneous analysis of neutron and X-ray scattering data. Biophys J 95(5):2356-2367

Kucerka N, Nieh M, Katsaras J (2009) Asymmetric distribution of cholesterol in unilamellar vesicles of monounsaturated phospholipids. Langmuir ACS J Surf Colloids 25(23):13522-13527

Kučerka N, Nieh M, Pencer J, Harroun T (2007) The study of liposomes, lamellae and membranes using neutrons and X-rays. Curr Opin Colloid Interf Sci 12:17-22

Kucerka N, Pencer J, Sachs JN, Nagle JF, Katsaras J (2007) Curvature effect on the structure of phospholipid bilayers. Langmuir 23(3):1292-1299

Kurdi M, DeMello Walmor C, Booz George W (2005) Working outside the system: an update on the unconventional behavior of the renin-angiotensin system components. Int J Biochem Cell Biol 37(7):1357-1367

Labinjoh C, Newby DE, Dawson P, Johnston NR, Ludlam CA, Boon NA, Webb DJ (2000) Fibrinolytic actions of intra-arterial angiotensin ii and bradykinin in vivo in man. Cardiovasc Res 47(4):707-714

Leclerc PC, Auger-Messier M, Lanctot PM, Escher E, Leduc R, Guillemette G (2002) A polyaromatic caveolin-binding-like motif in the cytoplasmic tail of the type 1 receptor for angiotensin ii plays an important role in receptor trafficking and signaling. Endocrinology 143(12):4702-4710
Lowe BM, Smith DG (1973) Glass electrode measurements in deuterium oxide. Analy Lett 6(10):903-907

Ludtke SJ, He K, Heller WT, Harroun TA, Yang L, Huang HW (1996) Membrane pores induced by magainin. Biochem Cell Biol 35(43): 13723-13728

Lundquist A, Wessman P, Rennie Adrian R, Edwards K (2008) Melittin-lipid interaction: a comparative study using liposomes, micelles and bilayer disks. Biochim Biophys Acta 1778(10): 2210-2216

MacMahon S, Peto R, Cutler J, Collins R, Sorlie P, Neaton J, Abbott R, Godwin J, Dyer A, Stamler J (1990) Blood pressure, stroke, and coronary heart disease. Part 1 , prolonged differences in blood pressure: prospective observational studies corrected for the regression dilution bias. Lancet 335(8692):765-774

Mariani P, Casadio R, Carsughi F, Ceretti M, Rustichelli F (1997) Structural analysis of membranes from photosynthetic bacteria by sans. Europhys Lett 37(6):433-438

Mason PC, Gaulin BD, Epand RM, Wignall GD, Lin JS (1999) Small angle neutron scattering and calorimetric studies of large unilamellar vesicles of the phospholipid dipalmitoylphosphatidylcholine. Phys Rev E 59(3):3361-3367

Mavromoustakos T, Theodoropoulou E, Dimitriou C, Matsoukas JM, Panagiotopoulos D, Makriyannis A (1996) Interactions of angiotensin ii with membranes using a combination of differential scanning calorimetry and $31 \mathrm{p} \mathrm{nmr} \mathrm{spectroscopy.} \mathrm{Lett} \mathrm{Pept}$ Sci 3(4): $175-180$

Nagle JF, Tristram-Nagle S (2000) Structure of lipid bilayers. Biochim Biophys Acta 1469(3):159-195

Nallet F, Laversanne R, Roux D (1993) Modeling X-ray or neutronscattering spectra of lyotropic lamellar phases-interplay between form and structure factors. J Phys II 3(4):487-502

Neutel JM, Smith DHG, Weber MA (1999) Is high blood pressure a late manifestation of the hypertension syndrome? Am J Hypertens $12(12): 215 \mathrm{~S}-223 \mathrm{~S}$

NIH (2003) The seventh report of the joint national commitee on prevention, detection, evaluation, and treatment of high blood pressure. Technical report, US Department of health and human services

Pabst G, Grage S, Dannerpongratz S, Jing W, Ulrich A, Watts A, Lohner K, Hickel A (2008) Membrane thickening by the antimicrobial peptide pgla. Biophys J 95(12):5779-5788

Pachori AS, Huentelman MJ, Francis SC, Gelband CH, Katovich MJ, Raizada MK (2001) The future of hypertension therapy: sense, antisense, or nonsense?. Hypertens Res 37(2 Part 2):357-364

Pencer J, Hallett FR (2000) Small-angle neutron scattering from large unilamellar vesicles: an improved method for membrane thickness determination. Phys Rev E Stat Phys Plasmas Fluids Relat Interdiscip Topics 61(3):3003-3008

Pencer J, Mills T, Anghel V, Krueger S, Epand RM, Katsaras J (2005) Detection of submicron-sized raft-like domains in membranes by small-angle neutron scattering. Euro Phys J E Soft Matter 18(4):447-458

Pencer J, Nieh M, Harroun TA, Krueger S, Adams C, Katsaras J (2005) Bilayer thickness and thermal response of dimyristoylphosphatidylcholine unilamellar vesicles containing cholesterol, ergosterol and lanosterol: a small-angle neutron scattering study. Biochim Biophys Acta 1720(1-2):84-91

Petoukhov MV, Svergun DI (2006) Joint use of small-angle X-ray and neutron scattering to study biological macromolecules in solution. Euro Biophys J 35(7):567-576

Ruiz-Ortega M, Ruperez M, Esteban V, Egido J (2003) Molecular mechanisms of angiotensin ii-induced vascular injury. Curr Hypertens Rep 5(1):73-79

Schmiedel H, Almasy L, Klose G (2006) Multilamellarity, structure and hydration of extruded popc vesicles by sans. Euro Biophys $\mathbf{J}$ Biophy 35(3):181-189 
Seto GWJ, Marwaha S, Kobewka DM, Lewis RNAH, Separovic F, McElhaney RN (2007) Interactions of the australian tree frog antimicrobial peptides aurein 1.2 , citropin 1.1 and maculatin 1.1 with lipid model membranes: differential scanning calorimetric and fourier transform infrared spectroscopic studies. BbaBiomembranes 1768(11):2787-2800

Severcan F, Sahin I, Kazanc N (2005) Melatonin strongly interacts with zwitterionic model membranes-evidence from fourier transform infrared spectroscopy and differential scanning calorimetry. Bba-Biomembranes 1668(2):215-222

Shin YA, Yoo S (1996) Conformational study of angiotensin ii. Biopolymers 38(2):183-190

Striker Gary E, Praddaude F, Alcazar O, Cousins Scott W, MarinCastano Maria E (2008) Regulation of angiotensin ii receptors and extracellular matrix turnover in human retinal pigment epithelium: role of angiotensin ii. Am J Physiol-Cell $\mathrm{Ph}$ 295(6):C1633-C1646

Stuhrmann HB, Burkhardt N, Dietrich G, Jünemann R, Meerwinck W, Schmitt M, Wadzack J, Willumeit R, Zhao J, Nierhaus KH (1995) Proton-and deuteron spin targets in biological structure research. Nucl Inst Methods Phys Res A 356:124-132

Surewicz WK, Mantsch HH (1988) Conformational properties of angiotensin-ii in aqueous-solution and in a lipid environment-a fourier-transform infrared spectroscopic investigation. J Am Chem Soc 110(13):4412-4414

Svergun DI, Koch MHJ (2003) Small-angle scattering studies of biological macromolecules in solution. Reports Progress Phys 66(10):1735-1782
Taylor WR (1999) Hypertensive vascular disease and inflammation: mechanical and humoral mechanisms. Curr Hypertens Rep 1(1):96-101

Tung S, Lee H, Raghavan SR (2008) A facile route for creating "reverse" vesicles: insights into "reverse" self-assembly in organic liquids. J Am Chem Soc 130(27):8813-8817

Valensin G, Delfini M, Gaggelli E (1986) Selective 1h-nmr relaxation investigations of membrane-bound drugs in vitro. 2. angiotensin ii. Biophys Chem 24(1):25-31

Wang Y, Qiu D, Cosgrove T, Denbow ML (2009) A small-angle neutron scattering and rheology study of the composite of chitosan and gelatin. Colloids Surf B Biointerf 70(2):254-258

Westh P (2009) Experimental approaches to membrane thermodynamics. Soft Matter 5(17):3249-3257

WHO (2003) Diet, nutrition and the prevention of chronic diseases. Technical report, World Health Organization

Winter R (2002) Synchrotron X-ray and neutron small-angle scattering of lyotropic lipid mesophases, model biomembranes and proteins in solution at high pressure. Biochimica et Biophysica Acta (BBA)/Protein Struct Mol Enzymol 1595(1-2):160-184

Wyse BD, Prior IA, Qian H, Morrow IC, Nixon Susan, Muncke Cornelia, Kurzchalia Teymuras V, Thomas Walter G, Parton Robert G, Hancock John F (2003) Caveolin interacts with the angiotensin ii type 1 receptor during exocytic transport but not at the plasma membrane. J Biol Chem 278(26):23738-23746

Zoumpoulakis P, Daliani I, Zervou M, Kyrikou I, Siapi E, Lamprinidis G, Mikros E, Mavromoustakos T (2003) Losartan's molecular basis of interaction with membranes and at 1 receptor. Chem Phys Lipids 125(1):13-25 